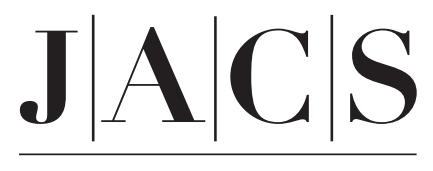

A R T I C L E S

Published on Web 12/04/2004

\title{
Self-Assembled Monolayers of Cobalt(II)- (4-tert-Butylphenyl)-Porphyrins: The Influence of the Electronic Dipole on Scanning Tunneling Microscopy Images
}

\author{
Valentina Arima, ${ }^{*}$ Eduardo Fabiano, Robert I. R. Blyth, Fabio Della Sala, \\ Francesca Matino, Julie Thompson, Roberto Cingolani, and Ross Rinaldi \\ Contribution from the National Nanotechnology Laboratory of INFM, Distretto Tecnologico \\ ISUFI, Università di Lecce, Via Arnesano, 73100 Lecce, Italy \\ Received May 18, 2004; E-mail: valentina.arima@unile.it
}

\begin{abstract}
Self-assembled monolayers (SAMs) of cobalt(II) 5,10,15,20-tetrakis(4-tert-butylphenyl)-porphyrin, a promising material for optical, photoelectrochemical, and chemical sensor applications, were prepared on $\mathrm{Au}(111)$ via axial ligation to 4-aminothiophenol, and studied by several surface science techniques. Scanning tunneling microscopy (STM) and spectroscopy (STS) measurements showed the apparent topology of the $\mathrm{Au}(111)$ herringbone structure reconstruction, but with bias-dependent contrast images and asymmetric $I / V$ characteristics. Photoelectron spectroscopy confirmed the presence of metalloporphyrins on the surface, whereas near-edge X-ray absorption (NEXAFS) measurements revealed that the porphyrin ring was tilted by about $70^{\circ}$ with respect to the surface plane. The above effects are ascribed to the presence of oriented molecular dipole layers between the metal and the organic material as confirmed by a comparison with first-principles density-functional theory calculations. The measured bias-dependent STM profiles have been reproduced by a simple monodimensional tunneling model.
\end{abstract}

\section{Introduction}

Ordered assemblies of metal tetraphenyl-porphyrins (MTPP) have recently attracted great interest for the formation of nanoscale molecular devices by virtue of their optical, photoelectrochemical, and chemical properties. For this reason, many ultrahigh vacuum scanning tunneling microscopy (UHV-STM) and spectroscopy (STS), ${ }^{1-5}$ photoemission $^{5,6}$ and near edge $\mathrm{X}$-ray absorption fine structure (NEXAFS) ${ }^{7}$ studies have been performed on evaporated metal porphyrins. STM measurements have always led to the identification of single porphyrin rings, clearly indicating that the tunneling probability is sensitive to electronic density of the molecular orbitals (essentially to the highest occupied and lowest unoccupied molecular orbitals, HOMO and LUMO, respectively). Moreover, photoemission and NEXAFS measurements have been used to investigate the occupied and unoccupied electronic states and to deduce the molecular orientation of the porphyrin ring on the surface. ${ }^{7}$

Although monolayers of physisorbed MTPPs have been wellstudied, chemisorbed self-assembled monolayers (SAMs) of metal-porphyrins have been studied only by surface-enhanced

(1) Scudiero, L.; Barlow, D. E.; Hipps, K. W. J. Phys. Chem. B 2000, 104, 11899-11905.

(2) Lu, X.; Hipps, K. W. J Phys. Chem. B 1997, 101, 5391-5396.

(3) Lu, X.; Hipps, K. W.; Wang, X. D.; Mazur, U. J. Am. Chem. Soc. 1996, 118, 7197-7202.

(4) Hipps, K. W.; Barlow, D. E.; Mazur, U. J. Phys. Chem. B 2000, 104, 24442447.

(5) Scudiero, L.; Barlow, D. E.; Mazur, U.; Hipps, K. W. J. Am. Chem. Soc. 2001, 123, 4073-4080.

(6) Scudiero, L.; Barlow, D. E.; Hipps, K. W. J. Phys. Chem. B. 2002, 106, 996-1003.

(7) Okajima, T.; Yamamoto, Y.; Ouchi, Y.; Seki, K. J. Elect. Spectrosc. Relat. Phenom. 2001, 114-116, 849-854.

10.1021/ja0470970 CCC: $\$ 27.50$ @ 2004 American Chemical Society
Raman scattering (SERS), ${ }^{8}$ electrochemical techniques, ${ }^{8}$ and UV/Vis spectroscopy. ${ }^{9}$ We have focused our attention on these SAMs because the preparation of the monolayer by wetprocessing is likely to be preferred to the relatively expensive and time-consuming evaporation method, in real device implementation.

In this work, we have studied a self-assembled monolayer of cobalt(II) 5,10,15,20-tetrakis(4-tert-butylphenyl)-porphyrin (CoTBPP) on gold(111), using STM and STS in UHV, photoelectron spectroscopy and NEXAFS. The aim of our work is to investigate, by comparing experimental and theoretical studies, how the molecules can be arranged and oriented on the surface and how their chemisorption can affect the electronic properties of the gold electrode.

To immobilize the porphyrin rings on the surface, we prepared a 4-aminothiophenol (4-ATP) self-assembled monolayer on gold(111) followed by axial legation in-situ of cobalt(II) 5,10,15,20-tetrakis(4-tert-butylphenyl)-porphyrin (CoTBPP).

STM is a well-established surface analysis technique. ${ }^{10,11}$ Although basic tunneling theory is well understood, the mechanism by which thin organic adsorbates (including physisorbed, chemisorbed, and Langmuir-Blodgett films) on conducting substrates are imaged is not yet unequivocally clarified. Studies

(8) Zhang, Z.; Hou, S.; Zhu, Z.; Liu, Z. Langmuir 2000, 16, 537-540.

(9) Kalyuzhny, G.; Vaskevich, A., Ashkenasy, G.; Shanzer, A.; Rubinstein, I J. Phys. Chem B 2000, 104, 8238-8244.

(10) Wiesendanger, R. Scanning Probe Microscopy and Spectroscopy; Cambridge University Press: Cambridge, 1994.

(11) Chen, C. J. Introduction to Scanning Tunneling Microscopy; Oxford University Press: Oxford, 1993. 
of long-chain alkanes, ${ }^{12-15}$ thiols, ${ }^{16-21}$ liquid crystals, ${ }^{22-24}$ and dialkyl-substituted benzenes ${ }^{6,22,25-28}$ on different conductive substrates, have demonstrated that image contrast can be controlled and modulated by changing the magnitude and polarity of the tip-sample bias.

Bias-dependent images, reported in such studies, indicate that there is a threshold bias at which the features of the monolayer can be discerned, thereby facilitating the observation of an adsorbate's electronic structure independent of the underlying substrate. When the threshold bias is achieved, the tunneling process through adsorbates can take place and contrast heights appear in the STM images. Two mechanisms have essentially been proposed to explain the origin of the STM image contrast: tunneling from molecular orbitals of the adsorbates (or resonant tunneling), ${ }^{22,29}$ and tunneling from a conductive substrate. ${ }^{15}$ In the first case, the frontier orbitals of the species participate in the tunneling process because the shape of the adsorbate, as imaged with the STM, approximately reflects the calculated electron density of the molecular orbitals. ${ }^{22,29}$ The second mechanism is often invoked to determine the nature of image contrast for electrically insulating adsorbates, e.g., simple and functionalized alkanes. Thibaudau and co-workers ${ }^{15}$ suggested that the contrast in STM images of $n$-alkanes on HOPG was dominated by elastic deformation of the substrate. Cyr et al. ${ }^{30}$ related the contrast of several functional groups $\left(\mathrm{NH}_{2}, \mathrm{CH}_{3}\right.$, $\mathrm{OH}, \mathrm{SH}, \mathrm{Cl}, \mathrm{Br}$, and I) in primary-substituted hydrocarbons to the local modulation of the substrate work function due to adsorbate polarizability. In their model, the presence of the polarizable layer induces a modification of the tunneling barrier and makes the physisorbed molecules "visible".

Furthermore, photoemission studies have shown the work function of the substrate can be modified by the addition of physisorbed or chemisorbed layers which introduce an oriented dipole. $^{31-34}$ This is true not only for chemisorbed layers, but

(12) Claypool, C. L.; Faglioni, F.; Matzger, A. J.; Goddard, W. A., III; Lewis, N. S. J. Phys. Chem. B 1999, 103, 9690-9699.

(13) Venkataraman, B.; Breen, J. J.; Flynn, G. W. J. Phys. Chem. 1995, 99, 6608-6619

(14) Driver, S. M.; Woodruff, D. P. Langmuir 2000, 16, 6693-6700.

(15) Thibaudau, F.; Watel, G.; Cousty, J. Surf. Sci. 1993, 281, L303.

(16) Qian, Y.; Yang, G.; Yu, J.; Jung, T. A.; Liu, G.-Y. Langmuir 2003, 19 6056-6065

(17) Nielsen, J. U.; Esplandiu, M. J.; Kolb, D. M. Langmuir 2001, 17, 34543459.

(18) Yamada, R.; Uosaki, K. Langmuir 2001, 17, 4148-4150.

(19) Kim, Y.-T.; McCarley, R. L.; Bard, A. J. J. J. Phys. Chem. 1992, 96, 74167421.

(20) Rosink, J. J. W. M.; Blauw, M. A.; Geerligs, L. J.; Van der Drift, E.; Rousseeuw, B. A. C.; Radelaar, S.; Sloof, W. G.; Fakkeldij, E. J. M. Langmuir 2000, 16, 4547-4553.

(21) Hara, M.; Sasabe, H.; Knoll, W. Thin Solid Films 1996, 273, 66-69.

(22) Lee, H. S.; Iyengar, S.; Musselman, I. H. Langmuir 1998, 14, 7475-7483.

(23) Stevens, F.; Dyer, D. J.; Müller, U.; Walba, D. M. Langmuir 1996, 12 $5625-5629$

(24) Walba, D. M.; Stevens, F.; Parks, D.; Clark, N. A.; Wand, M. D. Science 1995, 267, 1144-1147.

(25) France, C. B.; Schroeder, P. G.; Forsythe, J. C.; Parkinson, B. A. Langmuir 2003, 19, 1274-1281

(26) Qiu, X.; Wang, C.; Yin, S.; Zeng, Q.; Xu, B.; Bai, C. J. Phys. Chem. B. 2000, 104, 3570-3574.

(27) Lackinger, M.; Griessl, S.; Heckl, W. M.; Hietschold, M. J. Phys. Chem B. 2002, 106, 4482-4485.

(28) Rabe, J. P.; Buchholtz, S. Phys. Rev. Lett. 1991, 66, 2096-2099.

(29) Lindsay, S. M.; Sankey, O. F.; Li, Y.; Herbst, C.; Rupprecht, A. J. Phys. Chem. 1990, 94, 4655-4660.

(30) Cyr, D.; Venkataraman, B.; Flynn, G. W.; Black, A.; Whitesides, G. M. J. Phys. Chem. 1996, 100, 13747-13759.

(31) Ishii, H.; Sugityama, K.; Ito, E.; Seki, K. Adv. Mater. 1999, 11, 605-625.

(32) Hill, I. G.; Rajagopal, A.; Kahn, A.; Hu, Y. Appl. Phys. Lett. 1998, 73 662-664.

(33) Campbell, I. H.; Rubin, S., Zawodzinski, T. A.; Kress, J. D.; Martin, R. L.; Smith, D. L.; Barashkov, N. N.; Ferraris, J. P. Phys. Rev. B 1996, 54, $14321-14324$. also for physisorbed molecules. ${ }^{35,36}$ Therefore, the intrinsic dipole and polarizability of molecules are important factors in determining the adsorbate's effect on the surface work function and hence the STM image contrast.

When an STM image is acquired, one or more of these mechanisms may operate depending both on the coupling between the energy levels of the adsorbate and the surface Fermi levels, and on the spatial overlap between the STM probe and the molecules oriented on the substrate. A crucial role in the enhanced contrast and in the measured topographic height is played by the orientation of the molecules with the respect to the surface. A molecule, or ordered arrays of molecules, given conformational freedom, can result in unexpected STM images because they can assume different orientations on the surface. Although the intrinsic electronic structure of the molecular layer is only slightly modified, the geometry of the tip-molecule spatial overlap changes completely.

Analysis of data from ex-situ prepared samples provides certain challenges for the interpretation of surface sensitive spectroscopies, in that the presence of impurities, both from the sample preparation process and from adventitous adsorbates, cannot be avoided. Analysis of the Au/4-ATP system using both conventional XPS ${ }^{20}$ and synchrotron radiation photoemission ${ }^{37}$ indicates the presence of both free and oxidized 4-ATP, in addition to 4-ATP bonded to Au. Detailed curve fitting of results from our Au/4-ATP samples indicates that only $30 \%$ of the molecules present are actually 4-ATP bonded to $\mathrm{Au}^{37}$. Therefore, interpretation of NEXAFS data in particular will not be as straightforward as is the case for samples of a single species adsorbed on a clean surface under ultrahigh vacuum.

\section{Experimental Section}

Chemicals and Solvents. To allow wet processing, we have synthesized a more soluble version of cobalt(II) 5,10,15,20-tetrakisphenyl-porphyrin (CoTPP), cobalt(II) 5,10,15,20-tetrakis(4-tert-butylphenyl)-porphyrin (CoTBPP), by following the standard procedure ${ }^{38}$ for the metal-free porphyrin and using cobalt(II) acetate to insert the cobalt into the ring. The resulting material was purified by column chromatography, giving a final purity of $\sim 99 \%$ (characterized by liquid chromatography-mass spectroscopy and UV-visible absorption spectroscopy). 4-aminothiophenol (4-ATP), 90\%, was purchased from Aldrich and used as received. Ethanol and chloroform used were analytical grade. Chloroform was purified by filtration through a column of basic alumina.

Formation of the CoTBPP SAM on Gold(111) (Au/4-ATP/ CoTBPP). We anchored CoTBPP to the Au(111) surface via axial ligation to a self-assembled monolayer of 4-aminothiophenol (4-ATP), by immersing an Au/4-ATP SAM into a chloroform solution of CoTBPP $\left(10^{-4} \mathrm{M}\right)$ for $18 \mathrm{~h}$. Further details of the immobilization procedure are described in ref 39 . The structures of the molecules are shown in Figure 1.

STM and STS Measurements. Soon after deposition, the sample was inserted in the ultrahigh vacuum chamber to minimize possible

(34) Koller, G.; Blyth, R. I. R.; Sardar, S. A.; Netzer, F. P.; Ramsey, M. G. Appl. Phys. Lett. 2000, 76, 927-929.

(35) Duschek, R.; Mittendorfer, F.; Blyth, R. I. R.; Netzer, F. P.; Hafner, J.; Ramsey, M. G.; Chem. Phys. Lett. 2000, 318, 43-48.

(36) Blyth, R. I. R.; Mittendorfer, F.; Hafner, J.; Sardar, S. A.; Duschek, R.; Netzer, F. P.; Ramsey, M. G. J. Chem Phys. 2001, 114, 935-942.

(37) Arima, A: Matino, F.; Thompson, J.; Cingolani, R.; Rinaldi, R.; Blyth, R. I. R.; submitted for publication.

(38) Adler, A. D.; Longo, F. R.; Shergalis, W. J. Am. Chem. Soc. 1964, 86, $3145-3149$.

(39) Arima, V.; Blyth, R. I. R.; Della Sala, F.; Del Sole, R.; Matino, F.; Mele, G.; Vasapollo, G.; Cingolani, R.; Rinaldi, R. Mater. Sci. Eng. C 2004, 24, $569-573$ 


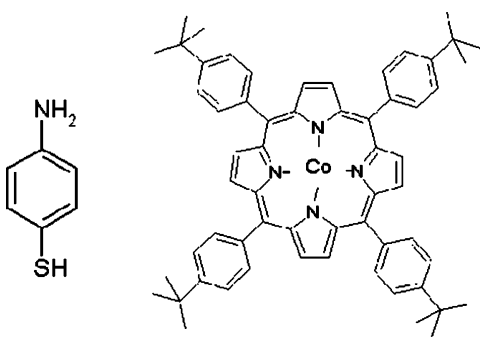

(a)

(b)

Figure 1. Molecular structures of (a) 4-aminothiophenol (4-ATP) and (b) Cobalt(II) 5,10,15,20-tetrakis(4-tert-butylphenyl)-porphyrin (CoTBPP).

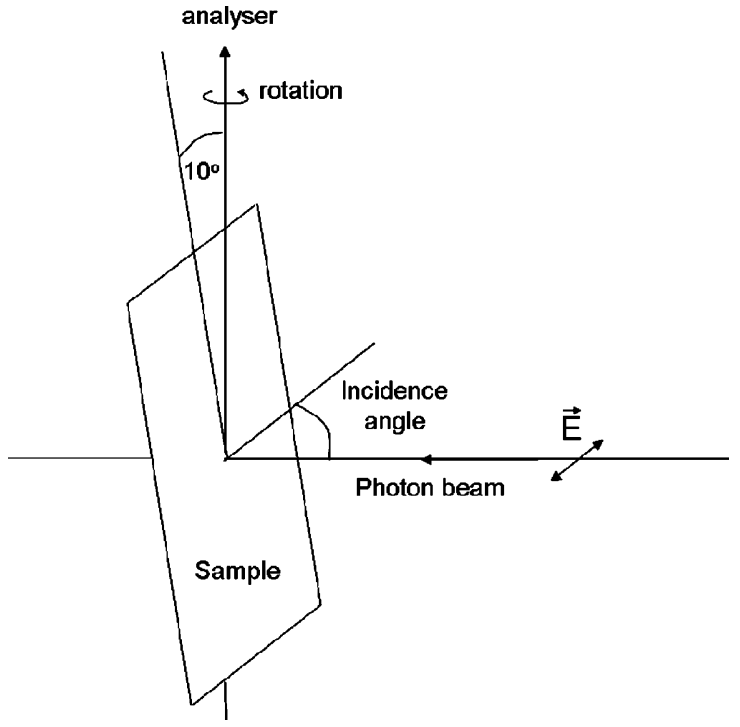

Figure 2. Experimental geometry for the photoemission and NEXAFS measurements.

contamination, which, however, cannot be avoided. The STM and STS measurements were carried out in UHV $\left(10^{-10} \mathrm{mbar}\right)$ at room temperature, using $\mathrm{Pt}-\mathrm{Ir}$ tips. Tip quality was tested by acquiring atomically resolved images of graphite. STM images were acquired in constant current mode using tip-biases ranging from \pm 0.2 to $\pm 1.5 \mathrm{~V}$ and a set point of $0.5-1 \mathrm{nA}$.

During spectroscopy measurements, the feedback loop was switched off and the set-point current, which sets the tip-sample distance, was fixed during the bias scan. The spectroscopy measurements $(I-V)$ were performed by collecting grids of points spaced by $6.25 \AA$ over scan areas of $50 \mathrm{~nm} \times 50 \mathrm{~nm}$. The STS spectra shown in this work are averaged over a set of several $I-V$ curves in the grid over the scanning area.

In addition, high rsolution contact mode atomic force microscopy (AFM) was also performed, in air, using a Thermomicroscopes CP Research Instrument AFM.

Photoemission and NEXAFS Measurements. Photoemission and NEXAFS spectra, at room temperature, were taken using the BEAR beamline $e^{40,41}$ at the ELETTRA synchrotron, Trieste, Italy. Samples were introduced into the vacuum system via a load lock. The sample mounting geometry used is shown in Figure 2. The analyzer was mounted vertically over the sample, which was mounted at a slight angle from the vertical. This gives high surface sensitivity, while the sample was rotated about the vertical axis to change the polarization of the incident light. Nitrogen 1s photoemission spectra were recorded

(40) Nannarone, S.; Borgatti, F.; DeLuisa, A.; Doyle, B. P.; Gazzadi, G. C.; Giglia, A.; Finetti, P.; Mahne, N.; Pasquali, L.; Pedio, M.; Selvaggi, G.; Naletto, G.; Pelizzo, M. G.; Tondello, G. AIP Conf. Proc. 2004, 705, 450.

(41) Borgatti, F.; De Luisa, A.; Doyle, B.; Giglia, A.; Mahne, N.; Pasquali, I. Pedio, M.; Selvaggi, G.; Nannarone, S.; Naletto, G.; Pelizzo, M. G.; Tondello, G. ELETTRA News, 2003, 47, September 30th. using a photon energy of $510 \mathrm{eV}$. A cylindrical mirror analyzer was used to acquire the photoemission spectra, which had an overall resolution (beamline plus analyzer) of $0.8 \mathrm{eV}$. Nitrogen K-edge NEXAFS spectra were recorded using Auger yield, with a resolution of $0.4 \mathrm{eV}$. Attempts were also made to obtain spectra of the Co $2 \mathrm{p}$ levels, however the relatively low flux at photon energies sufficient to excite the Co $2 p$ levels,${ }^{40,41}$ combined with the high background due to Au photoemission and Auger features, made meaningful data acquisition impossible.

\section{Theoretical Calculations}

We calculated the molecular geometry, binding energies and the electronic properties (dipole moment and polarizability) of the $\mathrm{Au} / 4$ ATP/CoTBPP assembly using a first-principles density-functional theory (DFT) approach. Calculations were carried out using the parallel quantum chemistry package TURBOMOLE. ${ }^{42}$ In the calculations, the 4-tert-butylphenyl substituent groups have been removed to reduce the computational cost, as they have a negligible effect on the electronic properties. The calculated system will be, hence, indicated as $\mathrm{Au} / 4$ ATP/CoP. Pseudopotentials ${ }^{43}$ were used to describe the gold core electrons. The resulting number of electrons is odd; therefore, an unrestricted DFT treatment was required.

For geometry optimization and energies, the gold substrate was simply modeled using a single gold atom, ${ }^{44}$ using the $\mathrm{TZVP}^{45-48}$ basis set and the B3LYP ${ }^{49}$ exchange-correlation (XC) energy functional.

For electronic properties, the Becke-Perdew ${ }^{50} \mathrm{XC}$ energy functional was employed together with the resolution-identity technique. ${ }^{51}$ The $\mathrm{Au}(111)$ substrate was modeled as a cluster of 21 gold atoms (in crystalline geometry) and oriented to form an angle of $70^{\circ}$ with the porphyrin plane (as obtained from NEXAFS measurements, see later). We checked that the number of gold atoms was sufficient to correctly describe the electronic properties. We note moreover that the choice of the XC functional influences the electronic dipole moment by less than $20 \%$. The sulfur atom was positioned in the hop hollow site, as recently described in ref 52 .

The electronic polarizability was computed using a finite-field of $10^{-4}$ a.u., and the dipole moment derived from Mulliken charges only on the molecule, i.e., without considering gold atoms. We note that the calculation of the electronic polarizability of a molecule attached to a metal cluster is completely dominated by the very large polarizability of the cluster, which moreover increases linearly with the number of metal atoms. ${ }^{53}$ This effect is not present in an open and semi-infinite gold substrate, like the one we have considered for the description of tunneling current.

\section{Experimental Results}

Bias-Dependent STM Images. Morphological characterizations of the clean gold(111) surface and of the 4-ATP monolayer, reported in ref 39, were performed before studying the CoTBPP monolayer. After deposition of the 4-ATP layer, the

(42) Ahlrichs, R.; et al., University of Karlsruhe, Germany, since 1988.

(43) Andrae, D.; Häusserman, U.; Dolg, M.; Stoll, H.; Preuss, H. Theor. Chim Acta 1990, 77, 123

(44) Derosa, P. A.; Seminario, J. M. J. Phys. Chem. B 2001, 105, 471-481.

(45) Schäfer, A.; Huber, C.; Ahlrichs, R. J. Chem. Phys. 1994, 100, 58295835.

(46) Schäfer, A.; Horn, H.; Ahlrichs, R. J. Chem. Phys. 1992, 97, 2571-2577 for hydrogen.

(47) Dunning, T. H. J. Chem. Phys. 1989, 90, 1007-1023 for polarization function.

(48) Woon, D. E.; Dunning, T. H. J. Chem. Phys. 1993, 98, 1358-1371.

(49) Becke, A. D. J. Chem. Phys. 1993, 98, 5648-5652.

(50) (a) Becke, A. D. Phys. Rev. A 1988, 38, 3098-3100; (b) Perdew, J. P. Phys. Rev. B 1986, 33, 8822-8824.

(51) Eichkorn, K.; Treutler, O.; Öhm, H.; Haser, M.; Ahlrichs, R. Chem. Phys Lett. 1995, 242, 652-660.

(52) Nara, J.; Higai, S.; Morikawa, Y.; Ohno, T. J. Chem. Phys. 2004, 120 6705-6711.

(53) Chandrakumar, K. R. S.; Ghanty, T. K.; Ghosh, S. K. J. Chem. Phys. 2004, $120,6487-6494$ 

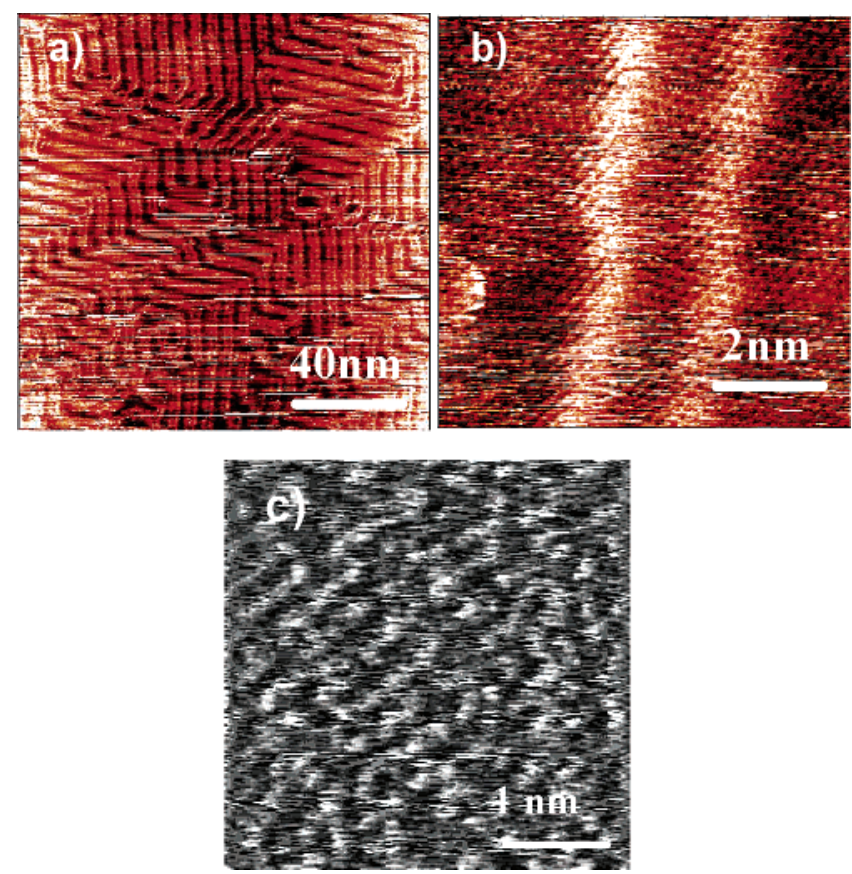

Figure 3. (a) Typical unfiltered STM images of Au/4-ATP/CoTBPP $(0.2$ $\mathrm{V}, 1 \mathrm{nA})$; (b) high-resolution STM image of Au/4-ATP/CoTBPP $(-1 \mathrm{~V}$, $0.5 \mathrm{nA}$ ); and (c) High-resolution AFM image of Au/4-ATP/CoTBPP.

gold terraces did not exhibit the characteristic pattern of Au$(111)-(22 \times \sqrt{ } 3)$ herringbone surface reconstruction, but rather exhibited a "web" pattern consisting of single and pairwise lines often linked by "U" connections. After the axial ligation of metalloporphyrins to the 4-ATP monolayer, the long range order of the $\mathrm{Au}(111)$ reconstruction, lost after 4-ATP adsorption, was recovered. In our previous work, ${ }^{39}$ we suggested that the observed phenomena were due to thiol adsorption which induced gold atom transport and modified the $\mathrm{Au}(111)$ surface morphology.

Figure 3a shows an STM image of the gold(111) surface after the CoTBPP deposition, recorded after positioning the tip close to the metal surface, i.e., by setting a relatively high tunneling current and low tip voltage. On the terraces, well-defined herringbone turns and pairwise-arranged parallel lines are clearly visible. The periodicity of the pairwise lines, whose nominal height is $0.15 \AA$, is $6.3 \mathrm{~nm}$, with $120^{\circ}$ bending on the herringbone turns, typical of the $\operatorname{Au}(111)-(22 \times \sqrt{ } 3)$ herringbone surface reconstruction. ${ }^{54}$

In an attempt to directly image the molecules, we gradually increased the tip bias to $0.8 \mathrm{~V}$; however, we did not observe any features which could be directly identified as molecules. High-resolution AFM, Figure 3c, does not indicate a pristine gold surface, but neither shows the well-resolved long range order seen in the STM images. However, the image shows the presence of structures which could be compatible with an ordered array of molecules, with a certain degree on conformational freedom, resulting in the loss of resolution as seen in Figure 3c.

When we applied a bias in the range +1 to $+1.5 \mathrm{~V}$ to the tip, we observed a strong and asymmetrical decrease of the apparent height of the pairwise-arranged lines, as shown in Figure 4a. From the line profile in Figure 4 (dotted line in Figure

(54) Barth, J. V.; Brune, H.; Ertl, G.; Behm, R. J. Phys. Rev. B 1990, 42, 93079318.
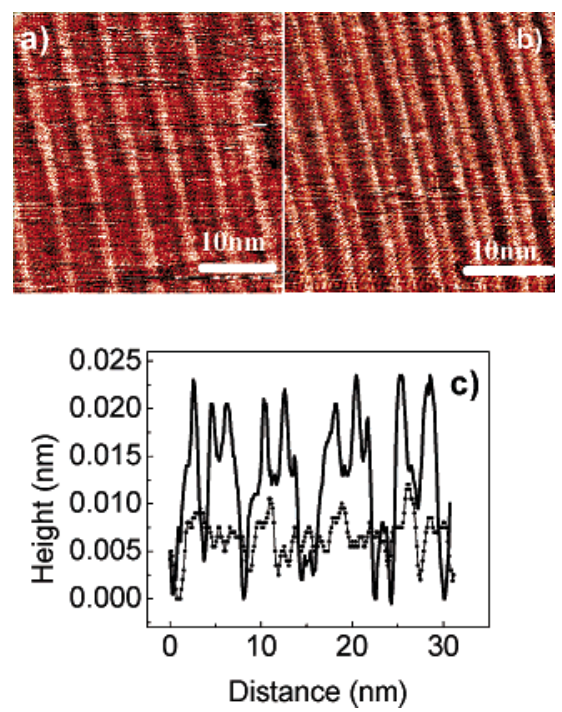

Figure 4. Typical unfiltered STM image of Au/4-ATP/CoTBPP at (a) 1.5 $\mathrm{V}, 0.2 \mathrm{nA}$; (b) $1.5 \mathrm{~V}, 0.5 \mathrm{nA}$; (c) line scan along the direction perpendicular to the $\mathrm{Au}(111)$ lines of Figure $4 \mathrm{a}$ (dotted line) and of Figure $4 \mathrm{~b}$ (solid line).
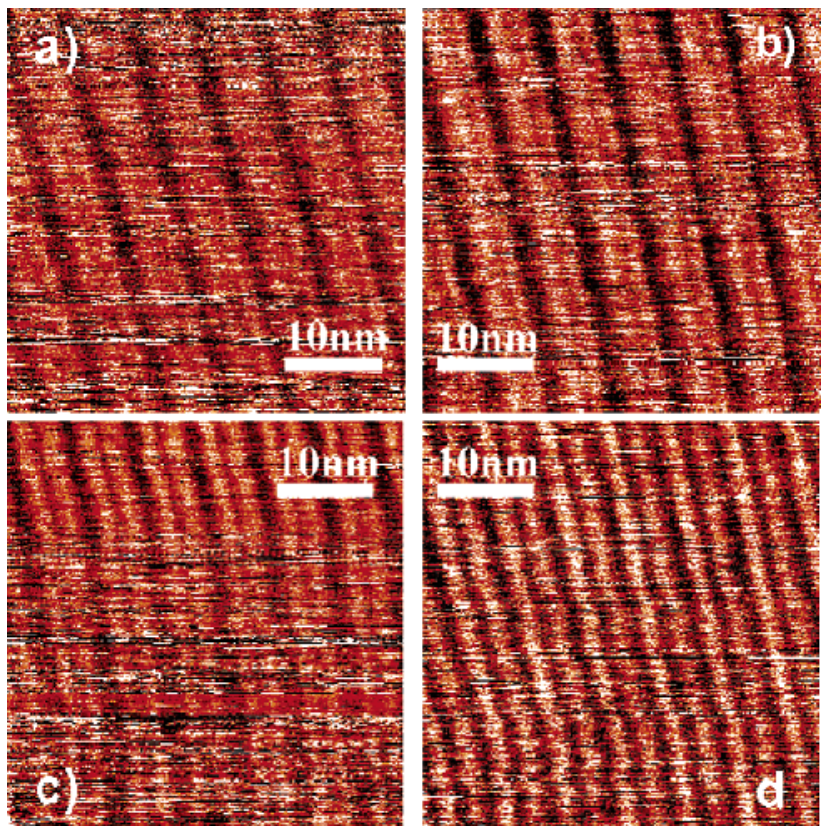

Figure 5. Typical unfiltered STM images of Au/4-ATP/CoTBPP at (a) $-0.2 \mathrm{~V}, 0.5 \mathrm{nA}$; (b) $-0.5 \mathrm{~V}, 0.5 \mathrm{nA}$; (c) $-0.8 \mathrm{~V}, 0.5 \mathrm{nA}$; (d) $-1 \mathrm{~V}, 0.5$ nA.

4c) it can be seen that the apparent height of one of the lines is $0.09 \AA$, whereas that of the other is $0.02 \AA$ lower. By further reducing the tip-sample distance, i.e., by increasing the current set point, the typical dimensions of the $\mathrm{Au}(111)-(22 \times \sqrt{ } 3)$ herringbone surface reconstruction were restored, as shown in Figure $4 \mathrm{~b}$ (solid line in Figure 4c). The highest positive bias at which we collected data was $+1.5 \mathrm{~V}$, since higher voltages were considered likely to cause molecular damage. Attempts to reduce the tip-surface distance, without modifying the applied voltage, led to loss of resolution.

We also observed a modulation of the $\mathrm{Au}(111)$ image by changing the tip-bias from -0.2 to $-1 \mathrm{~V}$, keeping the current set point fixed at $0.5 \mathrm{nA}$, as shown in Figure 5. The relatively broad raised lines seen in Figure 5a, gradually become resolved on increasing the negative voltage of the tip, into the charac- 


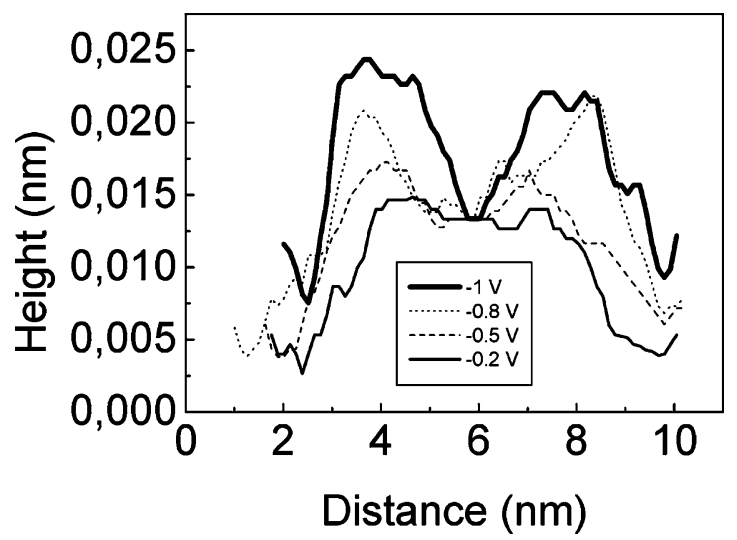

Figure 6. Line scan along the direction perpendicular to the $\mathrm{Au}(111)$ lines of Figure $5 \mathrm{a}-\mathrm{d}$.

teristic paired discommensuration lines of the $\mathrm{Au}(111)-(22 \times$ $\sqrt{ } 3$ ) reconstruction. As shown in Figure 5a, the profile of these discommensuration lines is strongly modified at low negative bias $(-0.2 \mathrm{~V})$, while it assumes the typical $\mathrm{Au}(111)$ profile at higher biases (max. values $-1 \mathrm{~V}$ ), as shown in Figure 5d. While the negative voltage is increasing, the line's apparent height, shown in Figure 6 (line profiles of figures 5a-d), gradually increases (from 0.1 to $0.17 \AA$ for one of the two lines) and the asymmetrical nature of the two lines becomes more visible (ranging from 0.01 to $0.03 \AA$ ). The central line becomes apparently deeper, ranging from 0.014 to $0.11 \AA$ (measured from the top of the higher line). Attempts to image the surface at higher negative bias again resulted in loss of resolution.

High-resolution images of the Au/4-ATP/CoTBPP surface, such as that shown in Figure 3b, exhibit the atomically resolved $\mathrm{Au}(111)-22 \times \sqrt{ } 3$ surface reconstruction. Note, however, that the asymmetry of the two lines still persists.

No bias-dependence was observed in images of the clean Au(111) substrate. Bias dependent measurements of the Au/4-ATP system ${ }^{37}$ showed only an increase in the apparent height of the discommensuration lines at higher voltages.

STS Measurements. STS spectra of clean gold and Au/4ATP/CoTBPP are shown in Figure 7. The clean gold samples show a symmetric $I(V)$ curve, whereas the Au/4-ATP/CoTBPP surfaces show asymmetric $I(V)$ curves with a higher current for negative sample bias. A quantitative estimate of the asymmetry of such curves (see the inset in Figure 7), the asymmetry grade, $k$, can be simply obtained from

$$
k=\frac{|I(V)|-|I(-V)|}{(|I(V)|+|I(-V)|) / 2}
$$

The inset to Figure 7 shows the asymmetry grades as a function of voltage derived from the data of Figure 7. From this it can be seen that for Au/4-ATP/CoTBPP there is a significant asymmetry, while for clean Au there is not.

Photoemission and NEXAFS Measurements. Flux normalized $\mathrm{N}$ 1s photoemission spectra of Au/4-ATP and Au/4-ATP/ CoTBPP are shown in Figure 8a. It is clear that for Au/4-ATP/ CoTBPP there is considerably more nitrogen present on the surface-comparing the areas of the peaks suggests a ratio of about $4: 1$. In principle, the coverage could be estimated using core level photoemission (i.e., comparing the N1s/Au4f ratio), but this measurement suffers from a high degree of uncertainty due to the inevitable presence of impurities - the measurement

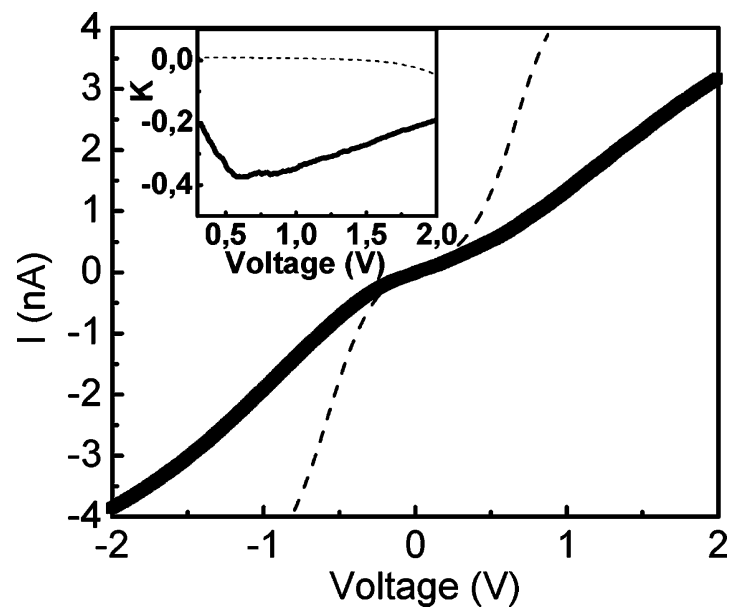

Figure 7. Scanning tunneling current - voltage curves (averaged over an area of $50 \times 50 \mathrm{~nm}$ ) recorded on different samples: clean gold (dashed line $-0.5 \mathrm{~V}, 0.5 \mathrm{nA}$ ); Au/4-ATP/CoTBPP (solid line $-1 \mathrm{~V}, 0.5 \mathrm{nA}$ ). Inset: asymmetry grade $(k=|I(V)|-|I(-V)| /(|I(V)|+|I(-V)|) / 2)$ of: clean gold (dashed line $-0.5,0.5 \mathrm{nA}$ ) and Au/4-ATP/CoTBPP (solid line $-1 \mathrm{~V}$, $0.5 \mathrm{nA})$ STS curves.
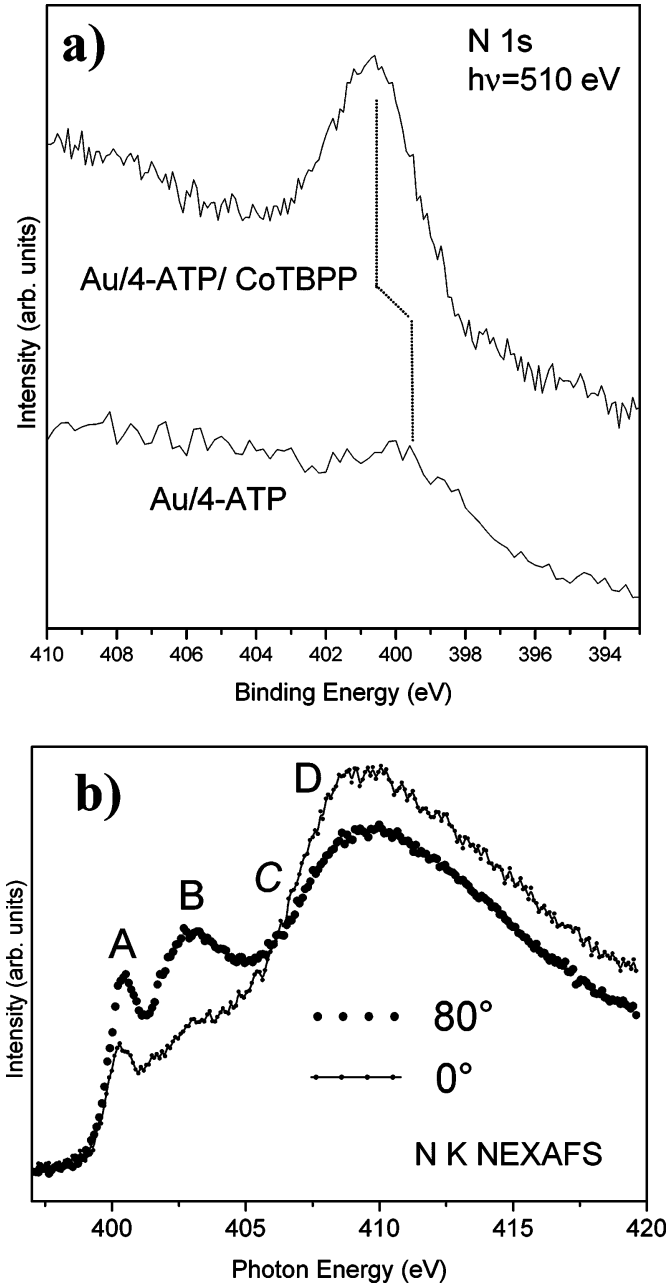

Figure 8. (a) Flux normalized $\mathrm{N}$ 1s photoemission spectra of Au/4-ATP and Au/4-ATP/CoTBPP; (b) Nitrogen K edge NEXAFS spectra of Au/4ATP/CoTBPP at grazing $\left(80^{\circ}\right)$ and normal $\left(0^{\circ}\right)$ incidence.

is not the usual calculation of the coverage of a surface species on an otherwise clean surface, but effectively that of a buried interface. We can perform a second order estimate by assuming that the coverage of 4-ATP is a monolayer, and comparing the 
flux-normalized intensities of the $\mathrm{N} 1$ s peak, as we have done in Figure 8a. This resulting 4:1 ratio suggests approximately monolayer coverage of the porphyrin.

The presence of only one $\mathrm{N}$ 1s peak in the Au/4-ATP/ CoTBPP in the data of Figure 8a confirms the presence of a metalloporphyrin, since metal-free porphyrin shows two peaks, due to protonated and unprotonated nitrogen. ${ }^{55}$ The binding energy of the porphyrin N1s peak is $400.6 \mathrm{eV}$, compared to 399.6 for that of 4-ATP. The value for 4-ATP is consistent with that of Rosinck et al. ${ }^{20}$ The value for CoTBPP is slightly higher than that of a neat CoTPP film, ${ }^{56}$ probably due to the reduced screening of the hole. A similar result was obtained by Da Cruz et al. ${ }^{56}$ when adsorbing CoTPP on silica via ligation to a siliconsubstituted pyridine.

Nitrogen K edge NEXAFS spectra of Au/4-ATP/CoTBPP are shown in Figure 8b. Reference spectra of metalloporphyrin evaporated films can be found in ref 7 . The low energy region of the NEXAFS spectra of metalloporphyrins consists of two peaks due to $\pi^{*}$ resonances, ${ }^{7}$ labeled $\mathrm{A}$ and $\mathrm{B}$ (following the notation of ref 7) in Figure 8b. A weak shoulder, due to a further $\pi^{*}$ resonance was also noted in ref 7 the approximate energy of this peak is indicated by the label " $C$ " in Figure $8 \mathrm{~b}$. The broader $\sigma$ peaks commence at around $407 \mathrm{eV}$, indicated by " $\mathrm{D}$ " in Figure 8b. The peaks are somewhat broadened compared to those of the evaporated Co porphyrin films, ${ }^{7}$ consistent with the results of Okajima et al. ${ }^{7}$ who noted a broadening in the spectra of $\mathrm{Co}$-porphyrin when exposed to NO, a molecule which would also be expected to bond to the porphyrin via the metal.

The $\pi^{*}$ resonances $\mathrm{A}$ and $\mathrm{B}$ in Figure $8 \mathrm{~b}$ show significant angular variation. Measuring the intensity ratio of the $\pi^{*}$ resonances in going from grazing to normal incidence, we obtain values of 1.3:1 and 1.6:1 for peaks $\mathrm{A}$ and $\mathrm{B}$, respectively. There is a significant contribution to the $\mathrm{N}$ NEXAFS signal due to the presence of the underlying 4-ATP layer. NEXAFS data from the Au/4-ATP system ${ }^{37}$ show little angular variation, as approximately $70 \%$ of this system is randomly oriented free and/ or oxidized 4-ATP. ${ }^{37}$ Therefore, the 4-ATP contrubutes a finite background at all angles to the data of Figure $8 \mathrm{~b}$, which we can estimate as around $20 \%$ of the total signal from a comparison of the N 1 s photoemission data of Figure 8a. We should expect that a small contribution to the variation of peak $\mathrm{B}$ is due to the intensity variation in this energy range from the underlying 4-ATP spectrum; ${ }^{37}$ therefore, it is not unexpected that the observed ratio is slightly higher for peak B compared to peak A. Taking the finite background into account, from the intensity plots of Stohr ${ }^{57}$ the observed intensity variations in the $\pi^{*}$ orbitals $\mathrm{A}$ and $\mathrm{B}$ correspond to angles of $65^{\circ}$ and $75^{\circ}$ relative to the surface. The intensity ratio of $\pi^{*}$ resonance NEXAFS is quite sensitive to small angular variations in this range ${ }^{56}$ therefore, the uncertainty is not particularly high.

\section{Theoretical Results}

The optimized molecular geometry of Au/4-ATP/CoP is shown in Figure 9. Interestingly, we found that the 4-ATP and porphyrin molecular planes are not orthogonal, but form an angle

(55) Polzonetti, G.; Ferri, A.; Russo, M. V.; Iucci, G.; Licoccia, S.; Paolesse, R. J. Vac. Sci. Technol. A 1999, 17, 832-839.

(56) Da Cruz, F.; Driaf, K.; Berthier, C.; Lamelle, J.-M.; Armand, F. Thin Solid Films 1999, 349, 155-161.

(57) Stöhr, J. NEXAFS Spectroscopy; Springer-Verlag: Berlin, 1992.

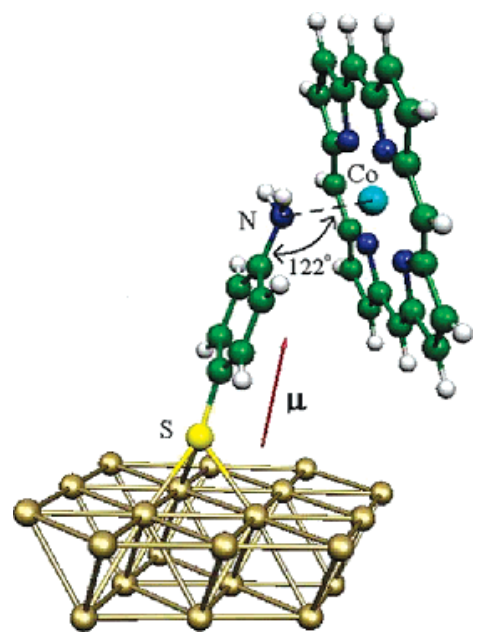

Figure 9. Optimized molecular geometry of Au/4-ATP/CoP. The arrow indicates the dipole moment.

of $122^{\circ}$. This is due to the tetrahedral hybridization assumed by the nitrogen atom. The computed $\mathrm{Co}-\mathrm{N}$ bond-length is 2.44 $\AA$. The binding-energy of the CoP to the Au/4-ATP has been computed as the total energy difference between the 4-ATP/ CoP optimized structure, and the system with the porphyrin and aniline well separated. The resulting energy is $49.50 \mathrm{kcal} / \mathrm{mol}$ $(2.15 \mathrm{eV})$, which shows that the $\mathrm{Co}-\mathrm{N}$ bond is quite strong.

The calculated HOMO-LUMO gap (using the B3LYP XC functional) of the 4-ATP/CoP is $3.01 \mathrm{eV}$. Both the HOMO and LUMO are $\pi$ orbitals localized in the porphyrin ring. Note that in this case HOMO and LUMO refer to the system 4-ATP/ CoP (i.e., without gold atoms).

The dipole moment of the Au/4-ATP/CoP assembly is 7.82 $\mathrm{D}$ and is indicated by the arrow in Figure 9. This dipole moment is mainly due to the 4-ATP molecule.

The static electronic polarization normal to the gold substrate is $267 \mathrm{au}$. This implies that the molecular electronic properties can be significantly modified by an external field, such as that of an STM tip.

\section{Discussion}

Although the presence of the CoTBPP SAM is confirmed by photoemission and NEXAFS measurements, it is completely invisible to the STM and appears electrically insulating in the limited voltage range that we have used (to avoid molecular damage). Therefore, to explain the experimental results in Figure 5 we cannot invoke the resonant tunneling mechanisms often used to describe STM images of $\pi$-electron rich layers. CoTBPP and similar molecules have been directly imaged with STM in conditions where the ring lies parallel to the surface. ${ }^{1-5}$ However, the conformational freedom induced by ligation via the 4-ATP, can result in a less conductive situation by permitting an adsorption geometry in which the spatial overlap of the tip wave functions with those of the molecular $\pi$ orbitals is much reduced. This would appear to be the situation in our case. From the results of the NEXAFS measurements, DFT calculations and molecular modeling we suggest that CoTBPP molecules are likely to be attached to the substrate as shown in Figure 10. This model takes into account the $70^{\circ}$ inclination of the porphyrin plane with respect to that of the substrate deduced from the NEXAFS spectra, and the $120^{\circ}$ calculated angle between the 4-ATP and the porphyrin, and also the presence of 
Tip

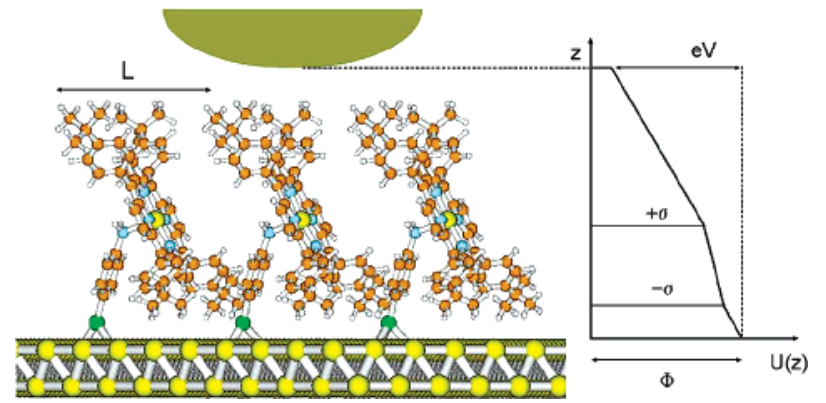

Figure 10. Model of the SAM. At right: schematic view of the potential used for the calculation of the trasmission coefficient between the tip and the substrate. $\Phi$ is the work-function of the substrate, $V$ is the applied bias and $\sigma$ is the surface charge density.

tert-butyl groups which have been found to determine how the porphyrin ring is rotated with respect to the $\mathrm{Co}-\mathrm{N}$ axis. With the orientation shown in Figure 10, the tert-butyls do not overlap with the substrate nor with the adjacent molecules, allowing a very close packing. In this configuration, the frontier orbital of the 4-ATP/CoTBPP system, which from DFT calculations we found located in the porphyrin ring, cannot spatially overlap with the STM probe molecular orbitals, due to the presence of tert-butyls, nor to the gold substrate, due to presence of 4-ATP. Therefore, no tunneling current through the molecular orbitals of the porphyrin ring is observed.

However, insulating monolayers can create voltage-dependent STM images if they introduce a dipole and are polarizable by the tip field: in this way, the substrate work function and the tunneling barrier can be modulated by the applied voltage. The DFT calculations show that the Au/4-ATP/CoTBPP system has a large dipole moment and polarizability. From Figure 9, it can be seen that the molecular dipole projection onto the substratenormal is almost complete. Moreover, experimental evidence of the presence of a molecular dipole is provided by the asymmetry of the STS measurements of Figure $7 .{ }^{58}$

In the energy range far from resonance with any molecular orbitals, the current is dominated by electron tunneling to the substrate through the molecule, which acts as a potential barrier. ${ }^{58,59}$ The current can be then expressed as

$$
I(V)=\frac{2 e}{h} \int_{0}^{e V} \rho_{t}(E-e V) \rho_{s}(E) T(E) d E
$$

where $\rho_{t}$ and $\rho_{s}$ are tip and substrate-molecule local densities of states (LDOS), $V$ is the applied bias and $T(E)$ is the transmission probability through the barrier. The substratemolecule LDOS shows in general peaks at molecular orbital energies but is flat within a small domain around the Fermi energy.$^{60}$ For low applied bias the substrate-molecule LDOS can thus be assumed constant, and consequently the current depends only on the transmission probability $T(E)$.

In a simple monodimensional model, which we use here, the transmission probability can be evaluated using the WKB

(58) (a) Rosink, J. J. W. M.; Blauw, M. A.; Geerlings, L. J.; Van der Drift, E.; Radelaar, S. Phys. Rev. B 2000, 62, 10459-10466; (b) Rosink, J. J. W. M.; Blauw, M. A.; Geerligs, L. J.; Van der Drift, E.; Rousseeuw, B. A. C.; Radelaar, S. Mater. Sci. Eng. C 1999, 8-9, 267-272.

(59) Samanta, M. P.; Tian, W.; Datta, S.; Henderson, J. I.; Kubiak, C. P. Phys. Rev. B 1996, 53, 7626-7629.

(60) Onipko, A. I.; Berggren, K.-F.; Klymenko, Y. O.; Malysheva, L. I.; Rosink, J. J. W. M.; Geerlings, L. J.; Van der Drift, E.; Radelaar, S. Phys. Rev. B 2000, 61, 11118-11124.
(Wentzel-Kramer-Brillouin) approximation

$$
T(E)=\exp \left[-\int_{0}^{z} d x \sqrt{\frac{8 m}{\hbar^{2}}(U(x)-E)}\right]
$$

where $z$ is the substrate-tip distance, $U(x)$ is the potential profile and $m$ is the electron effective mass, for which we have assumed $m=m_{e}$. The WKB method is known to strongly underestimate the transmission probability, ${ }^{61}$ nevertheless, it can be used to obtain a qualitative description in absence of resonances. The employed potential profile follows the molecular structure and is shown in Figure 10, where $\Phi$ represents the substrate and tip work functions and $\mathrm{eV}$ represents the applied voltage. For simplicity, as in ref 60 , the tip work function was assumed to be equal to that of the substrate $(5 \mathrm{eV})$ : small differences in barrier height do not have significant effects on transmission probability, as is pointed out in refs 58 and 62 . The permanent dipole of the molecule is approximated by the field of two parallel plane distributions (at distance $d=6 \AA$, i.e., the length of the aniline) of opposite charge density $\sigma$. Due to the high polarizability of the system the value of $\sigma$ actually depends on the applied voltage

$$
\sigma=\sigma_{0}+\alpha \frac{V}{z}=\sigma_{0}+\tilde{\alpha} V
$$

where $z$ is the tip-substrate distance, which in our case can be considered almost constant $(2 \mathrm{~nm})$ due to the very small tip movements (see Figure 6).

Equation 1 gives the $I-V$ characteristics in the presence of the molecular dipole. Equations 1 and 2 were computed by numerical integration and in order to obtain the tip-substrate distance, and thus to model STM line profiles, they can be solved for $z$, with fixed $I$ and $V$.

We have determined the values of $\sigma_{0}, \tilde{\alpha}$ in order to reproduce the experimental STM line profiles of Figure 6 and thus to determine in which region molecules are present. We divided the Au herringbone reconstruction into its three known distinct regions, ${ }^{54} \mathrm{~A}$ (FCC), B (Bridge) and C ( $\left.\mathrm{HCP}\right)$, as shown in Figure 11. STM contrast images (see Figure 6) suggest that CoTBPP molecules induce effects along the gold(111) discommensuration lines. In the model, values of $\sigma_{0}$ and $\tilde{\alpha}$ have been determined in each of the three regions, with region B closer to the tip than the others in order to follow the Au herringbone reconstruction. It turns out that the experimental STM profile can be very well reproduced assuming that no molecules are present in the $\mathrm{C}$ region, which then serves as a reference point. With $\sigma_{0}=0.006$ $\mathrm{e} / \AA^{2}$ and $\tilde{\alpha}=0.005 \mathrm{e} /\left(\AA^{2} \mathrm{~V}\right)$ in region $\mathrm{A}$ and reduced by a factor of 2 in the B region, the obtained STM profile is shown in Figure 12, which is in excellent agreement with Figure 6 as far as the voltage effects are concerned.

The obtained values of $\sigma_{0}$ and $\tilde{\alpha}$ can be directly related to the molecular dipole and polarizability of the 4-ATP/CoTBPP system, given an estimate of the molecular area. CoTBPP is 15 $\AA$ wide, thus the molecular area is $15 L \AA^{2}$, with $L$ given in Figure 10, which actually depends on the molecular coverage. With close packing, $L$ is about $1 \mathrm{~nm}$, thus the fitted values of $\sigma_{0}, \tilde{\alpha}$ correspond to a dipole of $25.4 \mathrm{D}$ and a polarizability of

(61) Cruz, E. L.; Helman, J. S. Phys Rev B 1974, 10, 1751-1754

(62) Dhirani, A.; Lin, P.-H.; Guyot-Sionnest, P.; Zehner, R. W.; Sita, R. J. Chem. Phys. 1997, 106, 5249-5253. 


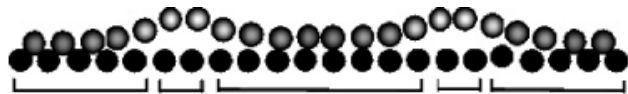 \\ $\mathrm{FCC}_{\text {Bridge }} \mathrm{HCP}$ Bridge $\mathrm{FCC}$}

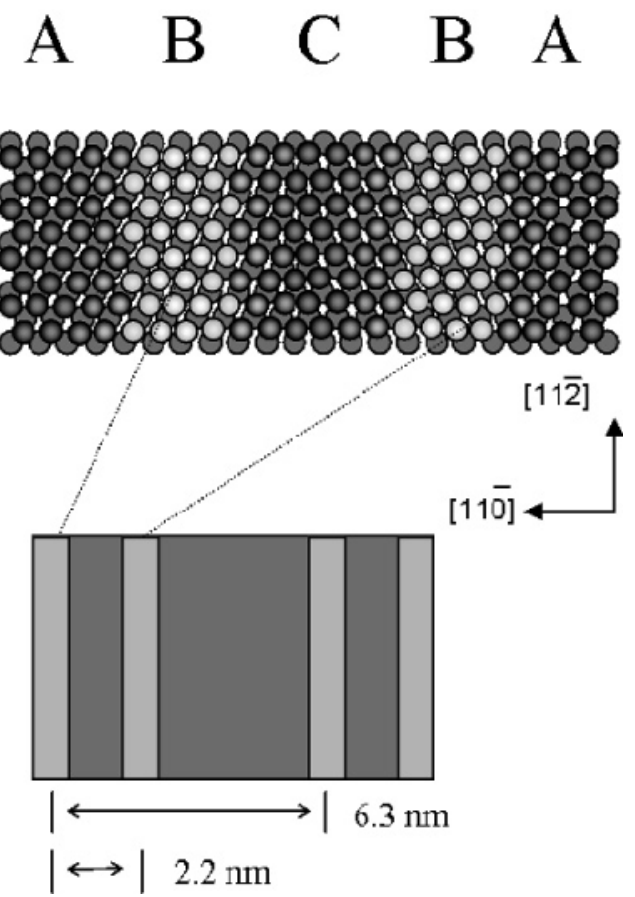

Figure 11. Schematic diagram of the parallel lines seen in Figure $3 b$, showing the geometric structure of the $\mathrm{Au}(111)-(22 \times \sqrt{3})$ surface reconstruction, lateral and top view.

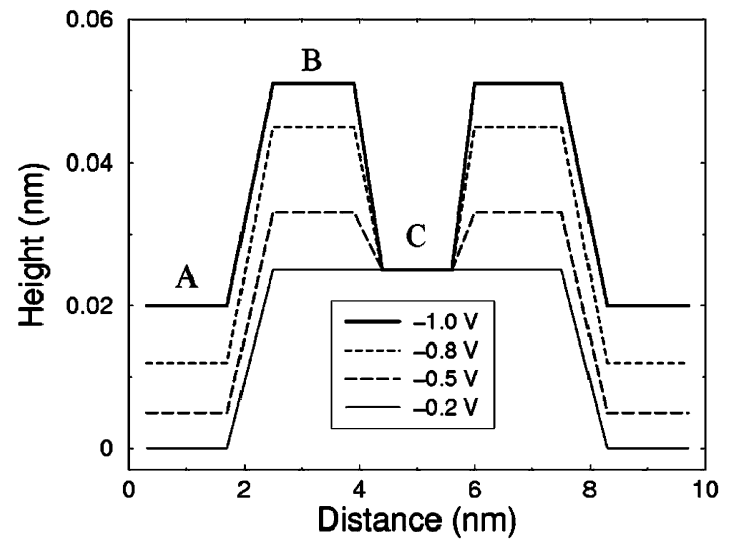

Figure 12. Calculated STM profile at different voltages along regions A,B,C.

308.9 a.u., which compare well with the values reported in the theoretical results section. Considering the employed WKB and monodimensional approximations in the tunneling model, the obtained value can be considered in excellent agreement with DFT results, suggesting that the obtained STM profile is due to the intrinsic molecular properties of the 4-ATP/CoTBPP system. Rows of closely packed 4-ATP/CoTBPP molecules are thus present in region $\mathrm{A}$. The reduced values of $\sigma_{0}$ and $\tilde{\alpha}$ in the $\mathrm{B}$ region, which is indeed important to reproduce the experi- mental profile, can be assigned to a reduced molecular coverage, probably due to the different organization of the gold surface near the discommensuration lines. ${ }^{54}$

These results can be used to discuss the STM profile of Figure 6. For negative tip bias, the electric field between the tip and the sample, and the dipole projection in the field direction, have opposite orientations. Moreover, the dipole projection in the field direction changes according to the applied voltage due to the high molecular polarizability. When the absolute value of the tip voltage is decreased, the effect of the dipole projection in the field direction is maximized. Therefore, when the negative tip voltage is decreased, the tunneling current decreases and the tip itself moves toward the surface in order to achieve the set point current.

The current decrease near bridge positions is slightly different if compared to the current decrease near the FCC sites because the molecular coverage and packing probably change near the discommensuration. For the above reasons, the traced profile is dependent on the voltage applied to the tip and has the shape shown in Figure 12. At low negative voltages $(-0.2 \mathrm{~V})$, the dipole effect is stronger and the traced profile is significantly different from the bare gold(111) line profile. When the voltage absolute value increases, the dipole effect slowly disappears and the resulting STM image reflects the structure of the gold surface. The asymmetrical nature of the two lines, just visible in the bias-dependent STM images (Figure 5) and particularly evident in the current-dependent STM images (Figure 4), can be ascribed to slightly different molecular packing of the 4-ATP/ CoTBPP system on the gold discommensuration lines.

\section{Conclusions}

The CoTBPP SAM has been studied by means of UHV STM and STS, photoemission spectroscopy, NEXAFS, and DFT calculations. We have demonstrated, by a comparison between experimental and theoretical results, that the chemisorbed molecules induced an electronic pertubation on the gold surface. The molecules, oriented as determined by NEXAFS, induced, in atomic resolved STM images of the $\mathrm{Au}(111)$ herringbone structure reconstruction, a contrast which varied as a function of tip-sample bias. STS measurements showed strong asymmetric characteristics which indicated the presence of an oriented dipole layer between the metal and the organic material. Theoretical studies on the molecular geometry and electronic properties were performed in order to elaborate a theoretical model of the STM image line profile, which was found to be in good agreement with the experimental one.

Acknowledgment. We thank the MIUR FIRB project "Molecular Devices" for funding and the BEAR staff, particularly Bryan Doyle, for assistance at ELETTRA. We thank R. Ahlrichs for providing the TURBOMOLE program package and G. Aloisio for his support. The calculations were carried on a $16 \mathrm{cpu}$ Compaq Alpha SC Server at CACT/ISUFI (Lecce). We thank the group of Prof. G. Vasapollo (NNL) for assistance with the synthesis of the CoTBPPs.

JA0470970 